\title{
亚胺氯化物介导一锅法合成 3-吸电子基团取代吲哚衍生物
}

\author{
占林俊 胡玮 王梅 黄斌 龙亚秋* \\ (苏州大学医学部药学院 苏州 215123)
}

\begin{abstract}
摘要 以 3-氰基/酯基吲哚为代表的 3-吸电子基团取代吲哚结构存在于多种生物活性分子中，常作为优势骨架构建类药 性化合物库用于新药发现. 本文设计并发展了一种基于亚胺氯化物活性中间体的串联反应，从邻卤苯乙腈/酯和酰胺出 发, 在碱和 $\mathrm{CuBr}$ 催化下，一锅法构建结构多样性 3-吸电子基团取代吲哚骨架的新合成方法. 该反应原料简单易得、反 应快速(5 min 完成)、产率高、底物适用范围广并可克级放大，具有原子经济性和步骤经济性，是基于优势骨架的多样 性导向的合成方法学(privileged substructure-based DOS)案例的重要补充.
\end{abstract}

关键词 一锅法合成; 亚胺氯化物; 3-吸电子基团取代吲哚; 类药性优势骨架

\section{Imidoyl Chloride Mediated One-Pot Synthesis of 3-Electron Withdrawing Group Substituted Indoles}

\author{
Linjun Zhan Wei Hu Mei Wang Bin Huang Ya-Qiu Long* \\ (College of Pharmaceutical Sciences, Soochow University, Suzhou 215123, China)
}

\begin{abstract}
Indole scaffold is widely present in pharmaceutical and pesticide products, dyes and natural products. The indole skeleton substituted by the electron withdrawing group at position 3 is an important class of bioactive indole derivatives. Among them, 3-cyanoindole is a key module in the construction of privileged scaffold-based combinatory library and diversity-oriented synthesis for drug discovery. For these reasons, the design and synthesis of these scaffolds have received considerable attention in organic synthesis and have been extensively studied. However, the cyano group on the indole was introduced directly in previously reported methods. Due to the high toxicity of cyanide, the application of these reactions is limited. Imidoyl chloride, a highly reactive synthon, which was successfully used as a module for the construction of quinolones, quinazolines, benzimidazoles and other drug-like privileged scaffolds by our group. By making use of the imidoyl chloride as the active intermediate to mediate a cascade reaction to form the heterocycle, we developed a new one-pot synthesis to construct the 3-cyano or carboxylate-indole derivatives. The reaction proceeded via two sequential steps: initial formation of imidoyl chloride starting from $N$-substituted arylamide and thionyl chloride, followed by 2-bromo-arylnitrile carbanion nucleophilic addition, elimination and Ulmann reaction. This synthetic methodology is featured with cheap and readily available starting materials, high reaction yields, high functional group tolerance and broad substrate scope. This reaction is a direct synthesis not requiring prefunctionalization, and highly atom- and step-economic. It's worth noting that it's the first time building 3-cyanoindole scaffold commenced with the substrates bearing cyano group, which is of great importance for avoiding potential safety hazards.
\end{abstract}

Keywords one-pot synthesis; imidoyl chloride; 3-electron withdrawing group substituted indoles; drug-like privileged scaffold

\section{1 引言}

吲哚骨架广泛存在于医药和农药产品、染料和天然 产物中, 是活性的重要结构载体, 被称为类药性优势骨 架 ${ }^{[1]}$. 其中, 3 位吸电子基团取代的吲哚骨架是吲哚衍生 物的重要类别 [2], 如 3-氧基吲哚和 3-酯基吲哚骨架存在 于许多活性分子中 [3], 诸如雌激素受体配体、丙型肝炎 抑制剂和 EZH2 抑制剂等(代表性结构见图 1). 同时, 3氰基吲哚结构在有机合成中有重要作用. 氰基可以转化 成一系列官能团, 包括醛基、胺、脒、四唑、酰胺及羧 基等, 是药物合成、材料化学和精密化学中的多功能骨 架 ${ }^{[4]}$. 抑制剂、次黄嘌呤核苷酸脱氢酶抑制剂、黄嘌呤氧化酶

*E-mail: longyaqiu@suda.edu.cn; Tel.: 0512-65882275; Fax: 0512-65884028

Received June 5, 2021; published June 8, 2021.

Supporting information for this article is available free of charge via the Internet at http://sioc-journal.cn.

Project supported by National Natural Science Foundation of China (Nos. 81761128022, 21772214, 81903442), National Science \& Technology Major Project “Key New Drug Creation and Manufacturing Program”, China (No. 2018ZX09711002-006-004), Natural Science Foundation of Jiangsu Province (No. BK20190817).

项目受国家自然科学基金(Nos. 81761128022, 21772214, 81903442)、国家科技重大专项 “重大新药创制”课题(No. 2018ZX09711002-006-004)和江苏 省自然科学基金(No. BK20190817)资助. 


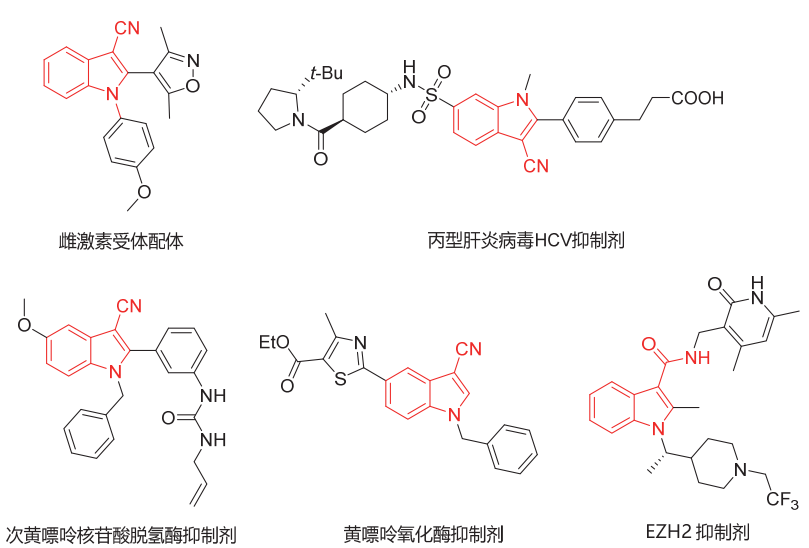

图 1 含 3-氰基/酯基吲哚骨架的代表性活性分子

Figure 1 Representative bioactive molecules bearing 3-cyano/carboxylate indole scaffolds

对于 3 位-氰基取代吲哚的合成, 此前报道的方法 主要采用在杂环上引入氧基的策略. 由于氧化物的剧毒 性, 主要的合成努力在于寻找氧基的替代物作为反应原 料. 例如, 王课题组 ${ }^{[5]}$ 在 2010 年报道了以 $\mathrm{K}_{4}\left[\mathrm{Fe}(\mathrm{CN})_{6}\right]$ 作为氰化试剂在吲哚 3 位引入氰基的反应; 该反应需要 加入催化量的醋酸钯和等物质的量的醋酸铜, 同时反应

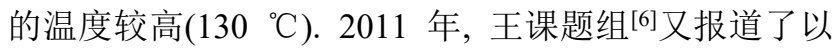
$\operatorname{NCTS}(N$-氧基- $N$-苯基对甲苯磺酰胺)为氧化试剂, 在路 易斯酸催化下在吲哚 3 位引入氧基的反应; 该反应以二 氯乙烷为溶剂, 反应条件较温和 $\left(80{ }^{\circ} \mathrm{C}, 12 \mathrm{~h}\right)$, 最高产率 可达 $97 \%$, 底物适用性也非常好, 只是反应的原子经济 性不强. 2012 年, Chang 课题组 ${ }^{[7]}$ 报道了铜催化的吲哚 3 位区域选择性氧基化反应, 该反应以碘化铵和 DMF 作 为氰基单元的组合源, 在 1 个大气压的氧气氛围里进行, 无需使用钯催化剂, 底物适用范围广, 具有良好的官能 团兼容性与区域选择性, 但反应温度高 $\left(130^{\circ} \mathrm{C}\right)$ 、反应时 间长 (12 h) 和操作不便. 2013 年, 吴课题组 ${ }^{88}$ 报道了钯催 化下异氮基化合物与 $N, N$-二甲基邻炔苯胺化合物形成 3-氰基吲哚的方法, 该反应条件温和、产率较高. 但底 物较难制备, 底物适用性差. 2020 年, 史课题组 ${ }^{[9]}$ 报道 了以 2-卤代苯胺和异恶唑-4-硼酸频哪醇酯作为原料, 通过钯催化串联反应快速构建 3-氰基吲哚的方法.

不同于在杂环底物上引进氰基的合成策略, 我们设 计直接从带有氰基的底物与活性中间体亚胺氯化物发 生串联反应构建氮杂芳环的 3-氰基吲哚合成方法, 以克 服氧基作为反应试剂所带来的反应局限性. 亚胺氯化物 是亚胺-烯胺的互变异构形式, 是一类反应活性较高的 合成子, 可以引发串联反应构建类药性优势骨架 ${ }^{[10]}$. 本 课题组前期应用亚胺氯化物为反应活性中间体，通过串 联反应高效便捷构建了喹诺酮 ${ }^{[11]}$ 、喹唑啉 ${ }^{[12]}$ 和苯并咪 唑 ${ }^{[13]}$ 等多种类药性优势骨架. 在此基础上, 本文设计以 邻卤苯乙腈(该结构类型的底物大多商业可售, 而且具 有丰富的取代基类型)为原料, 经与亚胺氯化物活性反 应子发生串联反应，一锅法合成 3-吸电子基团取代吲哚
衍生物(图 2), 其中亚胺氯化物活性中间体可由对应的 酰胺与二氯亚砜当场生成. 这也是首次报道采用直接带 有氰基的底物构建 3-氧基吲哚的反应策略，可与现有合 成方法形成互补，为构建结构多样性的氰基及其他吸电 子基团取代的杂芳环化合物提供更多的方法学选择，也 作为基于优势骨架的多样性导向的合成方法学 (privileged structure-based DOS)的重要补充.

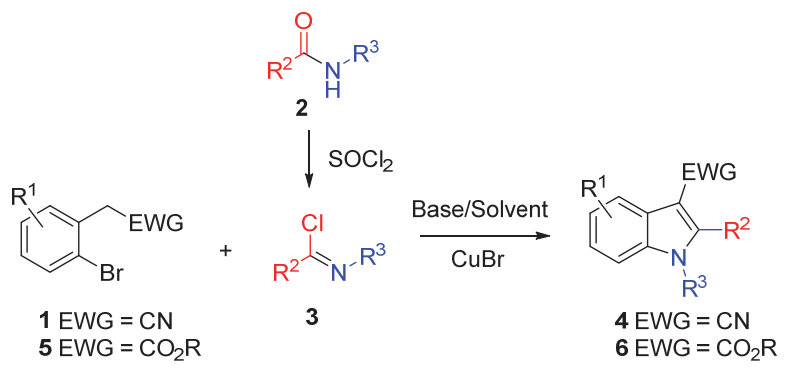

图 2 基于亚胺氯化物活性中间体介导串联反应的 3-吸电子基团取代 吲槑衍生物的一锅法合成

Figure 2 The imidoyl chloride mediated one-pot synthesis of 3-electron withdrawing group substituted indoles

\section{2 结果与讨论}

\section{1 反应条件的优化}

首先, 将 $N$-甲基苯甲酰胺 2a 在二氯亚砜中回流形 成相应的 $N$-甲基苯甲亚胺氯化物 3a. 我们以 2 -溴苯乙 腈 $\mathbf{1 a}$ 和 $N$-甲基苯甲亚胺氯化物 $\mathbf{3}$ 作为模板底物来篮选 反应的最优条件(如表 1 所示). 在常温下, 以 DMF 作溶 剂, 1a 与 3a 在 1.0 equiv. $\mathrm{NaH}$ 作用下反应 $5 \mathrm{~min}$, 随后将 温度升至 $80{ }^{\circ} \mathrm{C}$, 再加入 $\mathrm{K}_{2} \mathrm{CO}_{3}$ 与 $\mathrm{CuBr}$ 继续反应 $1 \mathrm{~h}$. 但 遗憾的是, 反应主要产出没有成环的 2-(2-溴苯基)-3-(甲 亚胺基)-3-苯代丙中间体( $\mathrm{C}-\mathrm{C}$ 偶联产物 4a'), 目标产 物 1-甲基-2-苯基-3-氧基吲哚 $\mathbf{4 a}$ 的产率仅为 $2 \%$ (表 1 序 列 1). 我们分离出未成环的中间体 $\mathbf{4 a}$ ', 分别针对 $\mathrm{NaH}$ 的用量、反应溶剂(1,4-二氧六环、DMF、DMSO)以及铜 催化剂种类 $(\mathrm{CuBr} 、 \mathrm{CuCl} 、 \mathrm{Cu}(\mathrm{OAc}) 、 \mathrm{CuI})$, 篎选了成 环反应条件(具体见 Supporting Information 表 S1), 发现 未成环的中间体 $\mathbf{4 a}$ '与 1.1 equiv. $\mathrm{NaH} 、 2$ equiv. $\mathrm{K}_{2} \mathrm{CO}_{3}$ 及 0.2 equiv. $\mathrm{CuBr}$ 在溶剂 DMF 中 $80{ }^{\circ} \mathrm{C}$ 下反应 $1 \mathrm{~h}$, 可以 充分转化为目标产物 $\mathbf{4 a}$. 在此基础上, 我们对于串联反 应的最优反应条件进行了考察. 我们推测 $\mathrm{NaH}$ 的用量 对目标化合物的产率影响较大, 于是对其进行了筛选 (表 1 序列 2 8), 发现 2.2 equiv. $\mathrm{NaH}$ 时产物 $\mathbf{4 a}$ 的产率 最高, 为 $84 \%$ (表 1 序列 7). 接着我们筛选了反应溶剂, 发现仍是 DMF 最优(表 1 序列 7), DMSO 和 1,4-二氧六 环的产率较低(表 1 序列 9 10). 我们进一步篮选不同的 反应温度对反应的影响, 发现升高或者降低温度都会降 低反应的产率(表 1 序列 11 12). 催化剂 $\mathrm{CuBr}$ 对反应 产率的影响也很大, 如果去除, 则目标化合物的产率急 剧下降(表 1 序列 13). 最终, 我们确定了一锅法合成 1甲基-2-苯基-3-氧基吲哚的最优条件为表 1 的序列 7, 即 
以 $\mathrm{DMF}$ 为溶剂, 底物 2-溴苯乙腈 $\mathbf{1 a}$ 在 $80{ }^{\circ} \mathrm{C}$ 下与 2.2 equiv. $\mathrm{NaH}, 2$ equiv. $\mathrm{K}_{2} \mathrm{CO}_{3}, 0.2$ equiv. $\mathrm{CuBr}$ 反应 5 min.

表 1 一锅法合成反应最优条件篮选 ${ }^{a}$

Table 1 Reaction conditions screening for the one-pot synthesis ${ }^{a}$

\begin{tabular}{|c|c|c|c|c|}
\hline 序列 & 溶剂 & $\mathrm{NaH}$ & $T /{ }^{\circ} \mathrm{C}$ & 产率 $b / \%$ \\
\hline 1 & DMF & 1.0 equiv. & 80 & 2 \\
\hline 2 & DMF & 1.2 equiv. & 80 & 8 \\
\hline 3 & DMF & 1.4 equiv. & 80 & 23 \\
\hline 4 & DMF & 1.6 equiv. & 80 & 53 \\
\hline 5 & DMF & 1.8 equiv. & 80 & 74 \\
\hline 6 & DMF & 2.0 equiv. & 80 & 77 \\
\hline 7 & DMF & 2.2 equiv. & 80 & 84 \\
\hline 8 & DMF & 2.4 equiv. & 80 & 82 \\
\hline 9 & DMSO & 2.2 equiv. & 80 & 43 \\
\hline 10 & 1,4-Dioxane & 2.2 equiv. & 80 & 11 \\
\hline 11 & DMF & 2.2 equiv. & 100 & 81 \\
\hline 12 & DMF & 2.2 equiv. & 60 & 67 \\
\hline $13^{[\mathrm{c}]}$ & DMF & 2.2 equiv. & 80 & 42 \\
\hline
\end{tabular}

a反应条件: $\mathbf{2 a}(2.2 \mathrm{mmol}), \mathrm{SOCl}_{2}(8.0 \mathrm{mmol}), \mathbf{1 a}(2.0 \mathrm{mmol}), \mathrm{K}_{2} \mathrm{CO}_{3}(4.0$ $\mathrm{mmol}), \mathrm{CuBr}(0.4 \mathrm{mmol})$, 溶剂 $(8 \mathrm{~mL})$, 反应时间为 $5 \mathrm{~min}$, 空气; ${ }^{b}$ 分离产 率; ${ }^{c}$ 无 $\mathrm{CuBr}$.

表 2 一锅法合成 3-氧基吲槑的底物范围 $a$

Table 2 Substrate scope for the one-pot synthesis of 3-cyanoindoles ${ }^{a}$

\section{2 底物适用性研究}

在确定了最优反应条件后, 我们对底物的适用性进 行了考察(表 2). 比较产物 $4 \mathrm{a} \sim 4 \mathrm{e}$ 的反应产率, 可以看 出，2-溴芳基乙腈芳香环上无论吸电子卤素取代或给电 子甲氧基取代，均能得到中等及以上收率(58\%～85\%), 其中给电子基团甲氧基取代收率较低(4e). 接着我们考 察了不同取代亚胺氯化物对反应收率的影响 $(\mathbf{4 f} \sim \mathbf{4 z}$, 4aa). 右侧苯环给电子甲氧基取代将降低反应收率(4f 与 4c); 相对于甲基取代, 氮原子异丙基取代时，产率下 降, 芐基取代时产率继续下降 $(\mathbf{4 c}, \mathbf{4 m}, 4 \mathrm{r})$, 有可能是因 为氮上位阻的因素影响关环，导致产率下降; $4 \mathbf{f}, 4 \mathrm{~g}, \mathbf{4 k}$, 40 可以看出同时有吸电子和给电子基团取代也具有中 等及以上收率(67\% 69\%). 4n 和 40 对比，同样说明吸 电子基团取代时反应优于给电子取代基. $4 \mathrm{p}$ 和 $4 \mathrm{y}$ 对比, 氮上有长链的梄链产率不如芳香环的取代, 氮上的给电 子因素增强也许对最后一步的关环脱卤素不利; $4 \mathrm{u}$ 和 $4 \mathrm{w}$ 中苯并呋喃和呋喃的不同芳香性对反应影响不大; 4x 中苯并噻吩在体系中也耐受; $4 \mathbf{a a}$ 中苯并吲哚中 2 位 是甲基取代也可以高收率得到产物.

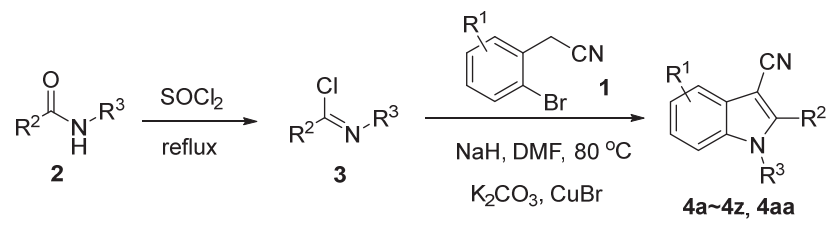

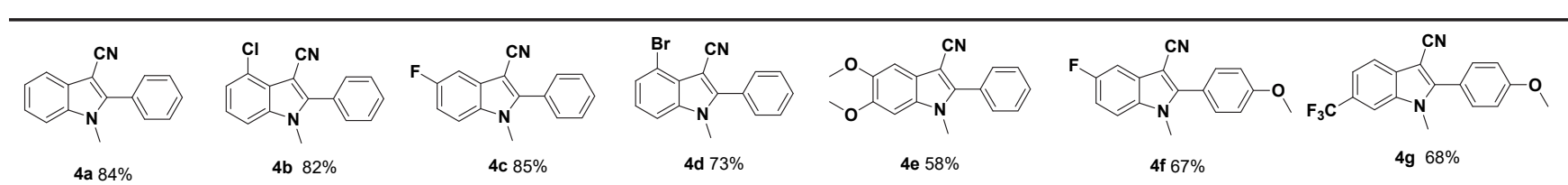

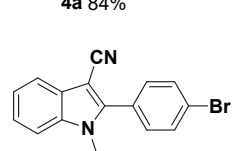

4h $78 \%$

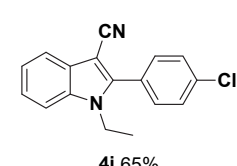

4d $73 \%$
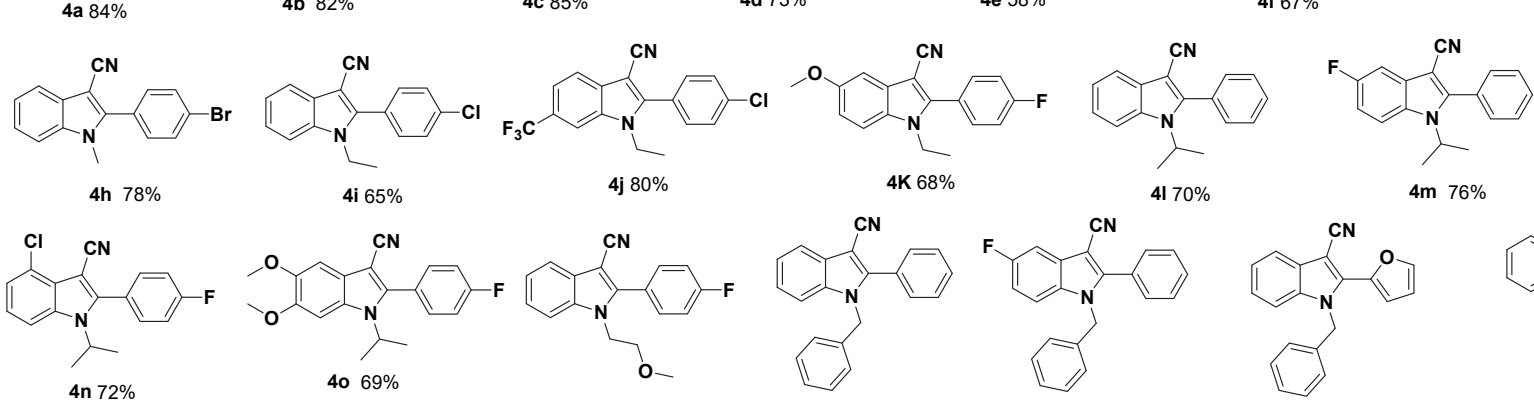

$\mathrm{N}$

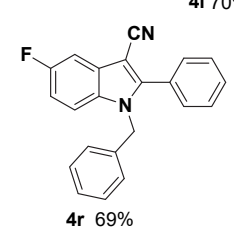

4m $76 \%$

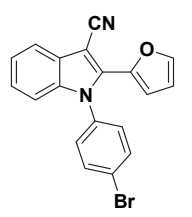

4u $63 \%$

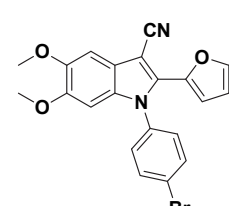

4v $57 \%$

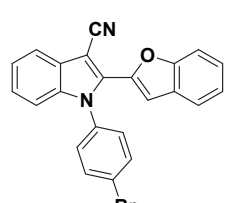

4w $64 \%$

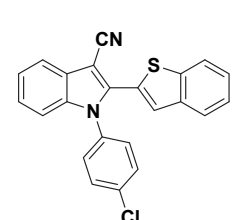

4x $61 \%$

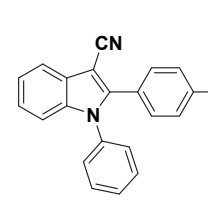

4y $68 \%$
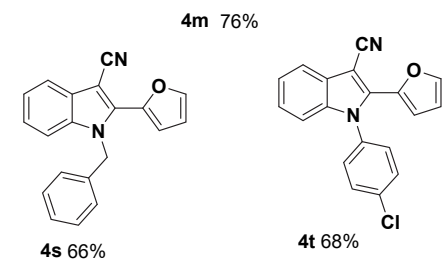

4s $66 \%$

${ }^{a}$ 反应条件: 1 (0.4 mmol), $3(0.44 \mathrm{mmol}), \mathrm{NaH}(0.88 \mathrm{mmol}), \mathrm{K}_{2} \mathrm{CO}_{3}(0.8 \mathrm{mmol}), \mathrm{CuBr}(0.08 \mathrm{mmol}), \mathrm{DMF}(8 \mathrm{~mL})$, 反应温度: $80{ }^{\circ} \mathrm{C}$, 反应时间: $5 \mathrm{~min}$. 
在成功建立亚胺氯化物介导的一锅法合成 3-氰基 吲哚的反应体系后, 我们将底物邻卤苯乙腈替换为邻卤 苯乙酯 5, 也能快速反应得到 3-酯基吲哚(表 3). 在生成 的 3-酯基吲哚中 $(\mathbf{6 a}, \mathbf{6 b}, \mathbf{6 c}, \mathbf{6 d})$, 左侧芳香环的电性因 素对产率影响不多, 都可达到较高收率; 从 $6 \mathrm{e}$ 看出, 右 侧苯环电性对产率影响不大. $6 \mathbf{f}$ 的氮上为乙基取代, 以 及右侧芳香环对于呋喃及苯并呋喃 $(6 \mathbf{h}, 6 \mathbf{6})$ 均耐受; $6 \mathbf{j}$ 的 高收率也可以用于脱苄基得到氮上无取代的酯基苯并 吲哚.

\section{表 3 一锅法合成 3-酯基吲梠的底物范围 $a$}

Table 3 Substrate scope for the one-pot synthesis of 3-carboxylate substituted indoles ${ }^{a}$

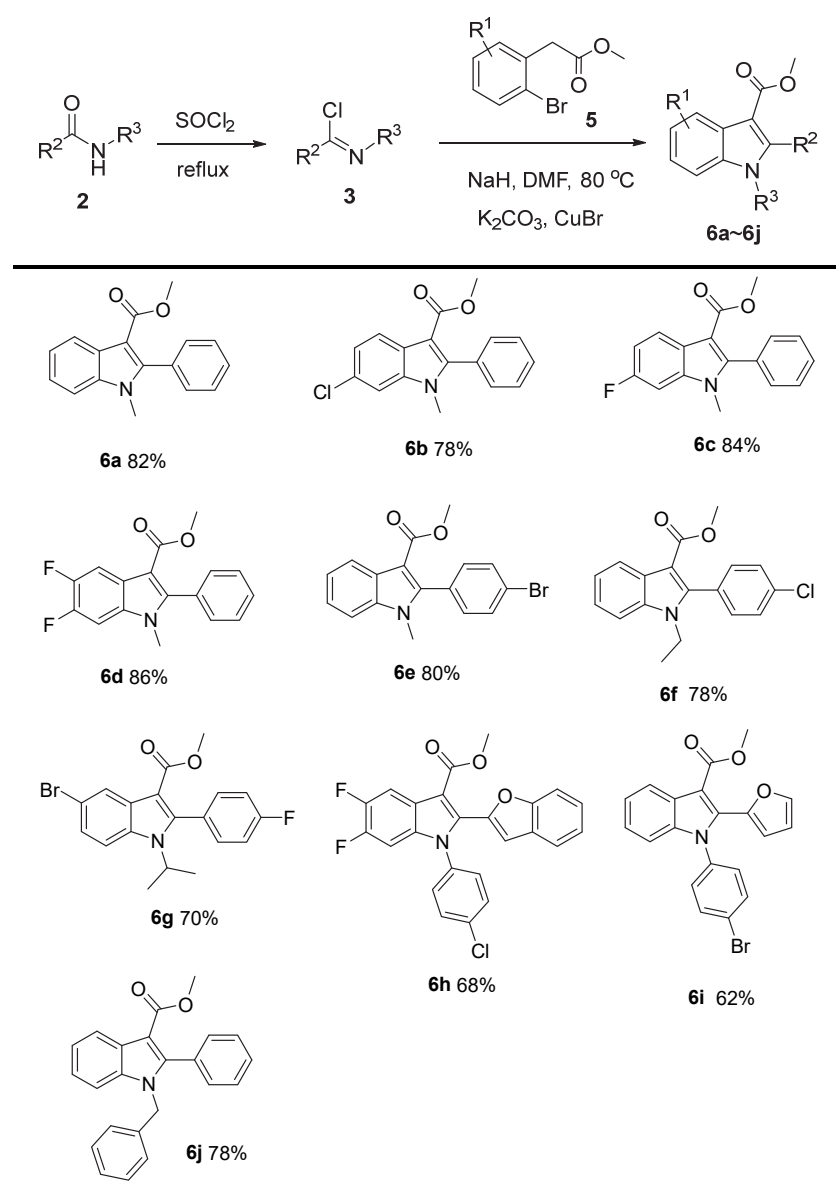

$a$ 反应条件: 5 (0.4 mmol), 3 (0.44 mmol), $\mathrm{NaH}(0.88 \mathrm{mmol}), \mathrm{K}_{2} \mathrm{CO}_{3}(0.8$ $\mathrm{mmol}), \mathrm{CuBr}$ (0.08 mmol), $\mathrm{DMF}(8 \mathrm{~mL})$, 反应温度: $80{ }^{\circ} \mathrm{C}$, 反应时间: $5 \mathrm{~min}$.
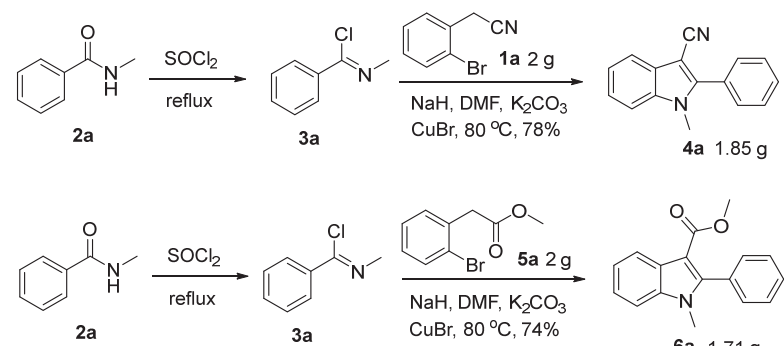

6a $1.71 \mathrm{~g}$

图 3 产物 $4 \mathrm{a}$ 和 $6 \mathrm{a}$ 的克级规模反应

Figure 3 The gram-scale synthesis of 3-cyanoindole $\mathbf{4 a}$ and 3-carboxylate indole $\mathbf{6 a}$
值得注意的是，该合成方法可以放大至克级规模 (图 3), 如 $2 \mathrm{~g}$ 的 $N$-甲基苯甲酰胺 $\mathbf{2 a}$ 在最优反应条件下 可以 78\%的分离产率得到 $1.85 \mathrm{~g}$ 产物 $4 \mathrm{a}$. 同样, $2 \mathrm{~g} \mathrm{~N}$ 甲基苯甲酰胺 $\mathbf{2 a}$ 在最优反应条件下也可以 $74 \%$ 产率得 到 $1.71 \mathrm{~g}$ 产物 $6 \mathrm{a}$, 说明该合成方法具有很强的实用性.

\section{3 可能的反应机理}

为了探索该反应的机理, 我们将一锅法反应分步进 行(图 4). 首先将 $N$-甲基苯甲酰胺通过二氯亚砜回流, 再加入 2-溴苯乙腈与 $\mathrm{NaH}$, 将反应中间体分离, 通过核 磁共振氢谱、碳谱与低分辨质谱确证了中间体的结构为 烯胺互变异构的中间体 4a'. 以此推定 2-溴苯乙腈被 $\mathrm{NaH}$ 篗取氢形成高活性的碳负离子，接着与 $N$-甲基苯 甲亚胺氯化物发生亲核取代反应得到该中间体 $\mathbf{4 a}$ '. 我 们将得到的中间体 $\mathbf{4 a}$ '在碳酸钾、氢化钠和溴化亚铜反 应条件下继续反应，可以得到相应的产物 $\mathbf{4 a}$.

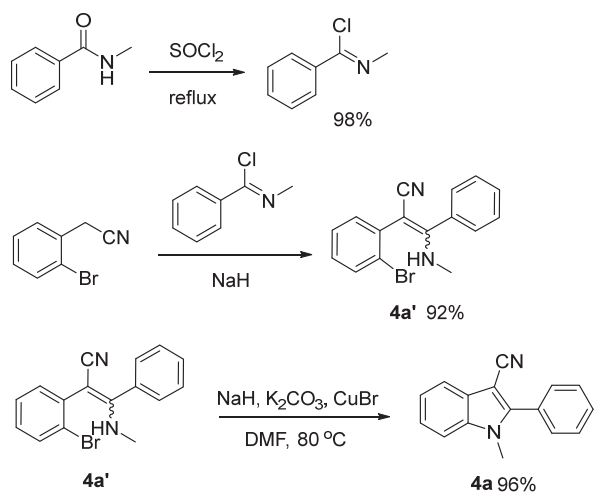

图 4 反应机理探索实验

Figure 5 Control experiments for the mechanism study

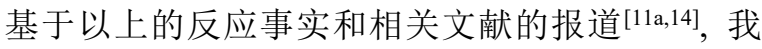
们提出了以下可能的反应机理(图 5). 首先是酰胺在二 氯亚砜回流下得到亚胺氯化物, 2-溴苯乙腈在钠氢的作 用下失去一个氢，形成了相应的碳负离子活性中间体. 该中间体与亚胺氯化物发生亲核加成-消除反应得到中 间体 $\mathbf{A}$, 该中间体亚胺在碱性条件下互变异构为烯胺 $\mathbf{B}$. 中间体 $\mathbf{B}$ 在碳酸钾、氢化钠和溴化亚铜反应条件下发生 分子内的乌尔曼偶联反应关环得到相应的吲哚产物.
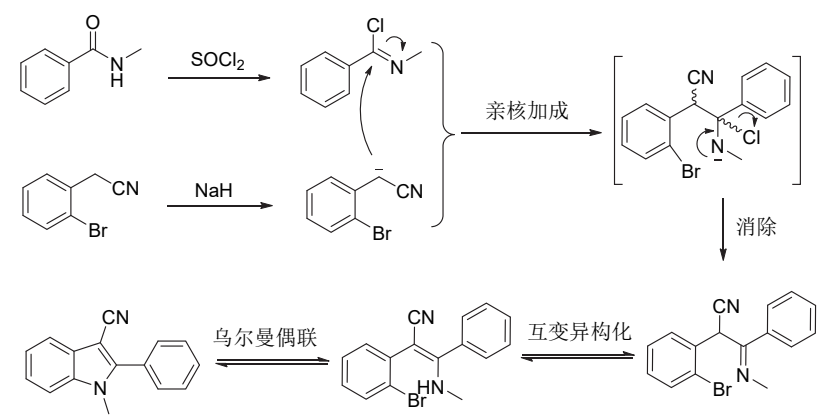

图 5 可能的反应机理

Figure 5 The proposed reaction mechanism 


\section{3 结论}

我们成功发展了一种以亚胺氯化物活性中间体介 导串联反应合成 3 位-吸电子基团取代吲哚衍生物. 以 价廉易得的底物邻溴苯乙腈与亚胺氯化物反应, 得到亲 核取代的亚胺中间体, 经过亚胺与烯胺互变异构及分子 内乌尔曼偶联反应关环得到吲哚环系, 产率最高可达 $85 \%$, 且反应条件温和, 反应 $5 \mathrm{~min}$ 内完成, 且底物适用 范围广. 原料邻卤苯乙腈, 取代基丰富、来源广泛、价 格便宜, 且直接带有氰基官能团, 避免了合成此类骨架 时引入氭基带来的安全隐患. 该反应体系也适用于 3 -酯 基吲哚的合成. 在实验操作上, 目标产物是由一锅法合 成, 无需分离中间体, 操作简便. 因此该反应方法学具 有安全、高效、高原子经济性等优点.

\section{4 实验部分}

一锅法合成 3-吸电子基团取代吲哚衍生物的实验 方法: 将 $N$-甲基苯甲酰胺(1.1 equiv.)溶于 $\mathrm{SOCl}_{2}$ 中, 氮 气氛围下回流摚拌反应 $2 \mathrm{~h}$. TLC 监测反应完成后, 迅速 用干燥的旋蒸除去溶剂(注: 空气潮湿, 水分较多, 会使 亚胺氯化物失效). 冰浴下将 2-溴苯乙腈(1 equiv.)溶于 干燥 DMF 中, 加入 60\% NaH (1.1 equiv.), 冰浴下搅拌 $15 \mathrm{~s}$ 后, 将上述制备的亚胺氯化合物加入到反应体系中 搅拌反应. 随后向反应体系加入碳酸钾 ( 2 equiv.), 溴化 亚铜(0.2 equiv.)和 $\mathrm{NaH}$ (1.1 equiv.), 并将反应温度升至 $80{ }^{\circ} \mathrm{C}$ 继续反应 $5 \mathrm{~min}$. TLC 监测反应进程, 反应结束后, 加入乙酸乙酯稀释反应液, 依次用饱和氯化铵溶液 (3 次)、饱和氯化钠溶液(3 次)洗涤, 收集有机相, 无水硫酸 钠干燥, 硅胶柱层析得目标产物 4a. 产物结构和纯度经 ${ }^{1} \mathrm{H}$ NMR, ${ }^{1} \mathrm{C}$ NMR, LC-MS 鉴定.

\section{References}

[1] (a) Cacchi, S.; Fabrizi, G. Chem. Rev. 2005, 105, 2873; (b) Crich, D.; Banerjee, A. Acc. Chem. Res. 2007, 40, 151; (c) Humphrey, G.
R.; Kuethe, J. T. Chem. Rev. 2006, 106, 2875.

[2] (a) Turner, D. R.; Chesman, A. S. R.; Murray, K. S.; Deacon, G. B.; Batten, S. R. Chem. Commun. 2011, 47, 10189; (b) Wade, L. G. J. Chem. Educ. 1991, 68, A86.

[3] (a) Swain, C. J.; Baker, R.; Kneen, C.; Moseley, J.; Saunders, J.; Seward, E. M.; Stevenson, G.; Beer, M.; Stanton, J.; Watling, K. J. Med. Chem. 1991, 34, 140; (b) Gu, X.-H.; Wan, X.-Z.; Jiang, B. Bioorg. Med. Chem. Lett. 1999, 9, 569; (c) Jiang, B.; Gu, X.-H. Bioorg. Med. Chem. 2000, 8, 363; (d) Wu, S.; Wang, L.; Guo, W.; Liu, X.; Liu, J.; Wei, X.; Fang, B. J. Med. Chem. 2011, 54, 2668; (e) Kumar, D.; Kumar, N. M.; Chang, K.-H.; Gupta, R.; Shah, K. Bioorg. Med. Chem. Lett. 2011, 21, 5897; (f) Murali Dhar, T. G.; Shen, Z.; Gu, H. H.; Chen, P.; Norris, D.; Watterson, S. H.; Ballentine, S. K.; Fleener, C. A.; Rouleau, K. A.; Barrish, J. C.; Townsend, R.; Hollenbaugh, D. L.; Iwanowicz, E. J. Bioorg. Med. Chem. Lett. 2003, 13, 3557; (g) Zhang, N.; Zhang, X.; Zhu, J.; Turpoff, A.; Chen, G.; Morrill, C.; Huang, S.; Lennox, W.; Kakarla, R.; Liu, R.; Li, C.; Ren, H.; Almstead, N.; Venkatraman, S.; Njoroge, F. G.; Gu, Z.; Clausen, V.; Graci, J.; Jung, S. P.; Zheng, Y.; Colacino, J. M.; Lahser, F.; Sheedy, J.; Mollin, A.; Weetall, M.; Nomeir, A.; Karp, G. M. J. Med. Chem. 2014, 57, 2121; (h) Rhoennstad, P.; Kallin, E.; Apelqvist, T.; Wennerstaal, M.; Cheng, A. WO 2009127686, 2009.

[4] (a) Demko, Z. P.; Sharpless, K. B. J. Org. Chem. 2001, 66, 7945; (b) Neumann, J. J.; Suri, M.; Glorius, F. Angew. Chem., Int. Ed. 2010 49, 7790; (c) Suri, M.; Jousseaume, T.; Neumann, J. J.; Glorius, F. Green Chem. 2012, 14, 2193; (d) Ueda, S.; Nagasawa, H. J. Am. Chem. Soc. 2009, 131, 15080; (e) Kuang, J.; Chen, B.; Ma, S. Org. Chem. Front. 2014, 1, 186.

[5] Yan, G.; Kuang, C.; Zhang, Y.; Wang, J. Org. Lett. 2010, 12, 1052.

[6] Yang, Y.; Zhang, Y.; Wang, J. Org. Lett. 2011, 13, 5608.

[7] Kim, J.; Kim, H.; Chang, S. Org. Lett. 2012, 14, 3924.

[8] Qiu, G.; Qiu, X.; Liu, J.; Wu, J. Adv. Synth. Catal. 2013, 355, 2441.

[9] Wu, J.; Liu, J.; Zhou, K.; He, Z.; Wang, Q.; Wu, F.; Miao, T.; Qian, J.; Shi, Q. Chem. Commun. 2020, 56, 12660.

[10] (a) Surgenor, R. R.; Grote, A. C.; McGeough, C.; Narayanan, A.; Wang, W.; Collier, P. N. J. Org. Chem. 2020, 85, 14158; (b) Wang, L.-C.; Du, S.; Chen, Z.; Wu, X.-F. Org. Lett. 2020, 22, 5567; (c) Chen, C.; Wang, Y.; Shi, X.; Sun, W.; Zhao, J.; Zhu, Y.-P.; Liu, L.; Zhu, B. Org. Lett. 2020, 22, 4097; (d) Chen, Z.; Wang, W.-F.; Yang, H.; Wu, X.-F. Org. Lett. 2020, 22, 1980; (e) Yang, H.; Lu, S.-N.; Chen, Z.; Wu, X.-F. J. Org. Chem. 2021, 86, 4361.

[11] (a) Lin, J.-P.; Long, Y.-Q. Chem. Commun. 2013, 49, 5313; (b) Wang, S. F.; Lin, J. P.; He, P. L.; Zuo, J. P.; Long, Y.-Q. Acta Chim. Sinica 2014, 72, 906 (in Chinese). (王沈丰，林建平，何佩岗，左建 平, 龙亚秋, 化学学报, 2014, 72, 906.)

[12] Cheng, Y.; Shen, J.; Peng, R.-Z.; Wang, G.-F.; Zuo, J.-P.; Long, Y.-Q. Bioorg. Med. Chem. Lett. 2016, 26, 2900.

[13] Lin, J.-P.; Zhang, F.-H.; Long, Y.-Q. Org. Lett. 2014, 16, 2822.

[14] (a) Jiao, J.; Zhang, X.-R.; Chang, N.-H.; Wang, J.; Wei, J.-F.; Shi, X.-Y.; Chen, Z.-G. J. Org. Chem. 2011, 76, 1180; (b) Kwong, F. Y.; Klapars, A.; Buchwald, S. L. Org. Lett. 2002, 4, 581.

(Yang, X.) 